\title{
FieldML - a meta-language for field interchange
}

Richard Christie, Poul Nielsen, Chris Bradley, Caton Little, Randall Britten, Peter Hunter

Auckland Bioengineering Institute

CellML workshop, Auckland, 11 April 2011 


\section{Requirements for FieldML}

- A standard format for interchanging field descriptions and data between different software.

- Able to describe fields of arbitrary complexity.

- Efficient.

- Extensible.

- Reusable model components. 


\section{Existing Field Formats}

Typical features:

- Fixed 'element types' combine shape and function

- 'Nodal' interpolation

- Special treatment of coordinates

- Unstructured or limited structured mesh support

- Large number of concepts

- Use standard field names to convey meaning

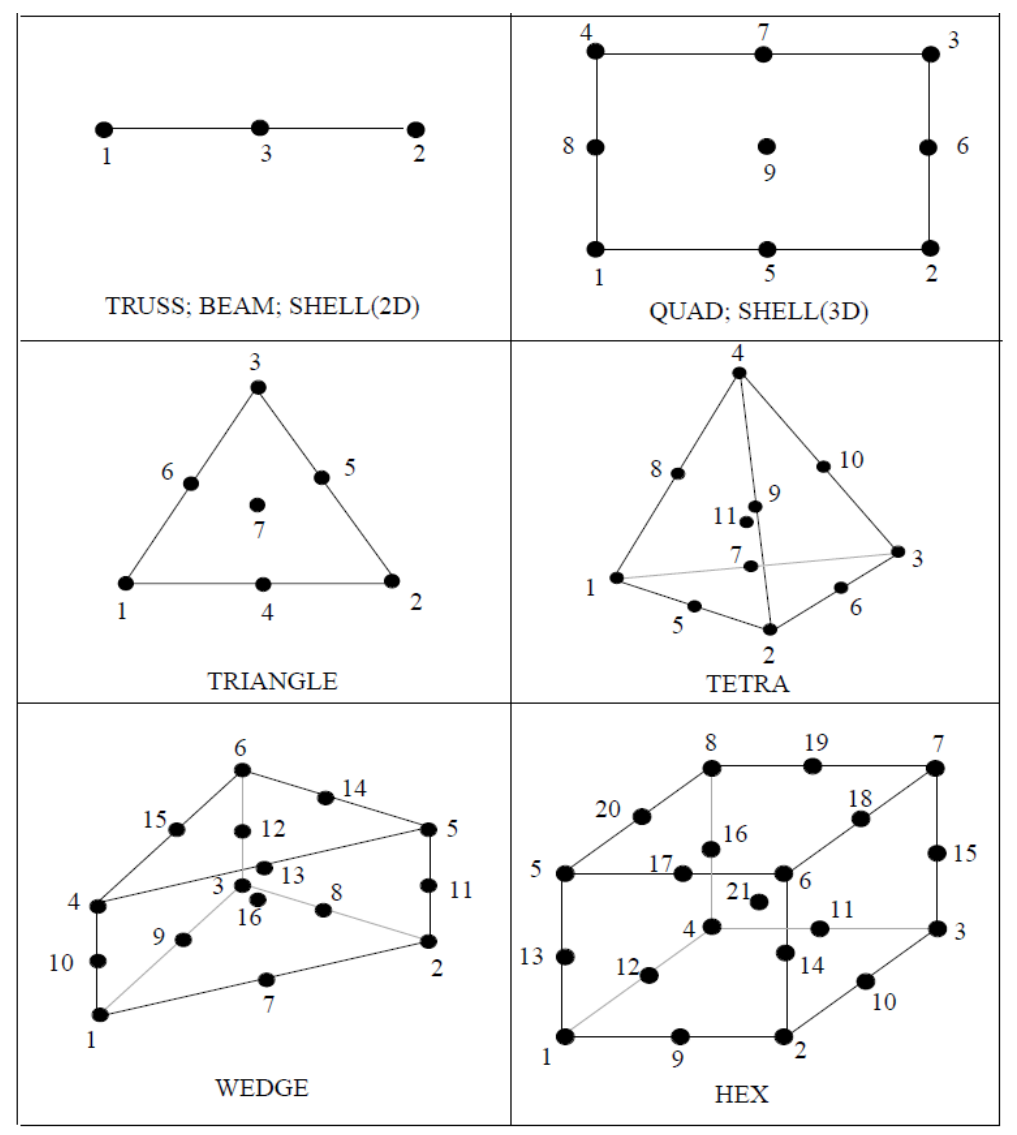

Exodus II format, Sandia National Labs 


\section{Background: CMISS/cmgui}

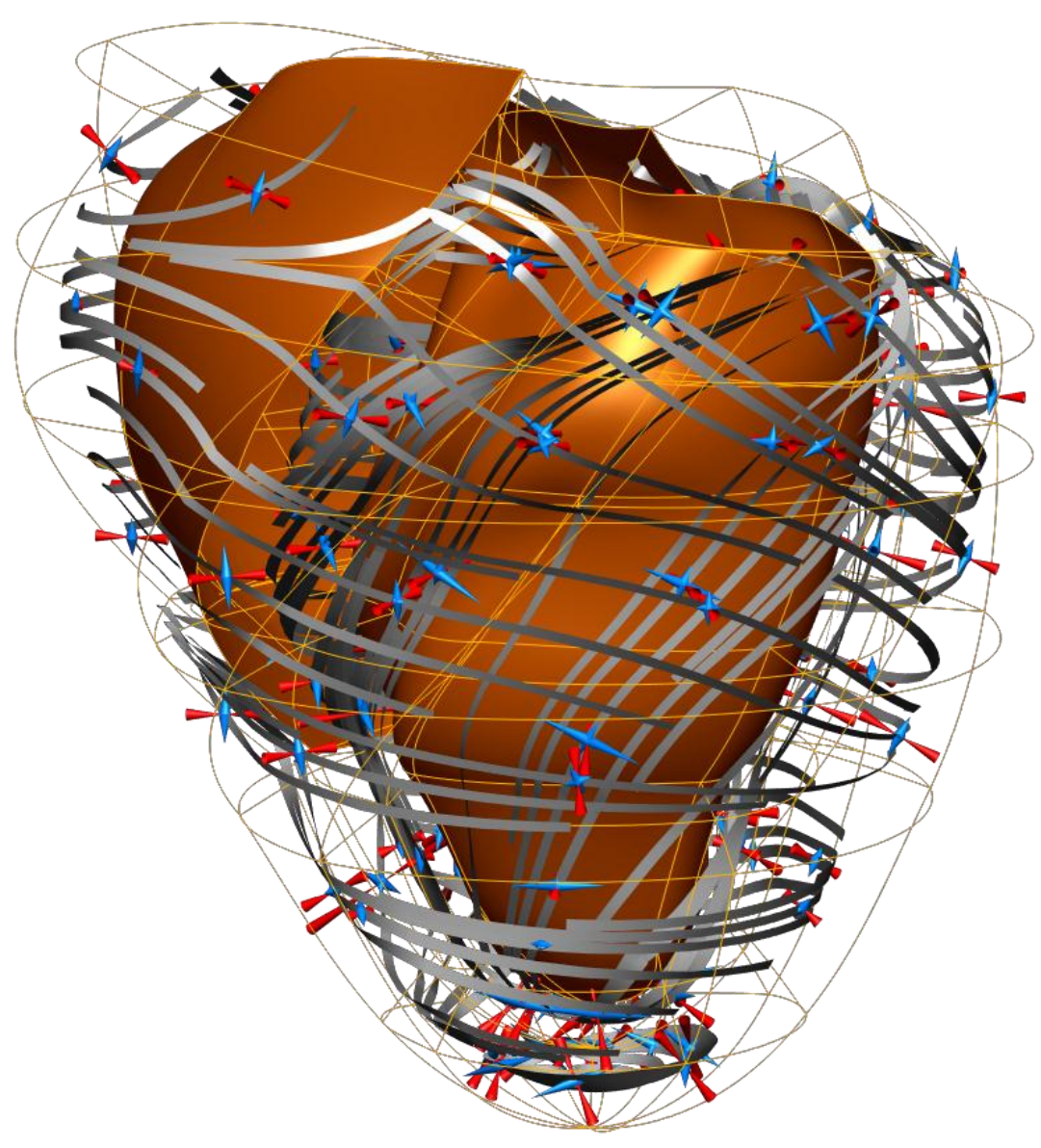

- 'Everything is a field': geometry, material properties, solution, derived values.

- Separation of shape and function.

- High order basis functions and complex parameter mappings.

- Time indexed parameters.

- Fields derived by mathematical expressions on other fields incl. composition/embedding.

- Image \& image processing fields.

BUT:

- Fixed function interpolation.

- Only linear time variation.

- Object overheads. 


\section{FieldML Design Philosophy}

- A meta-language for applications to describe their structures and data in a standard way.

- Base on minimal set of fundamental concepts.

- Avoid fixed, legacy structures esp. low-level objects, their limitations and overheads.

- Simple if simple, complex if complex.

- Preference for homogeneous data.

- Separation of bulk data from high-level description.

- Use metadata to communicate purpose of fields and specialised relationships. 


\section{Domains and Fields}

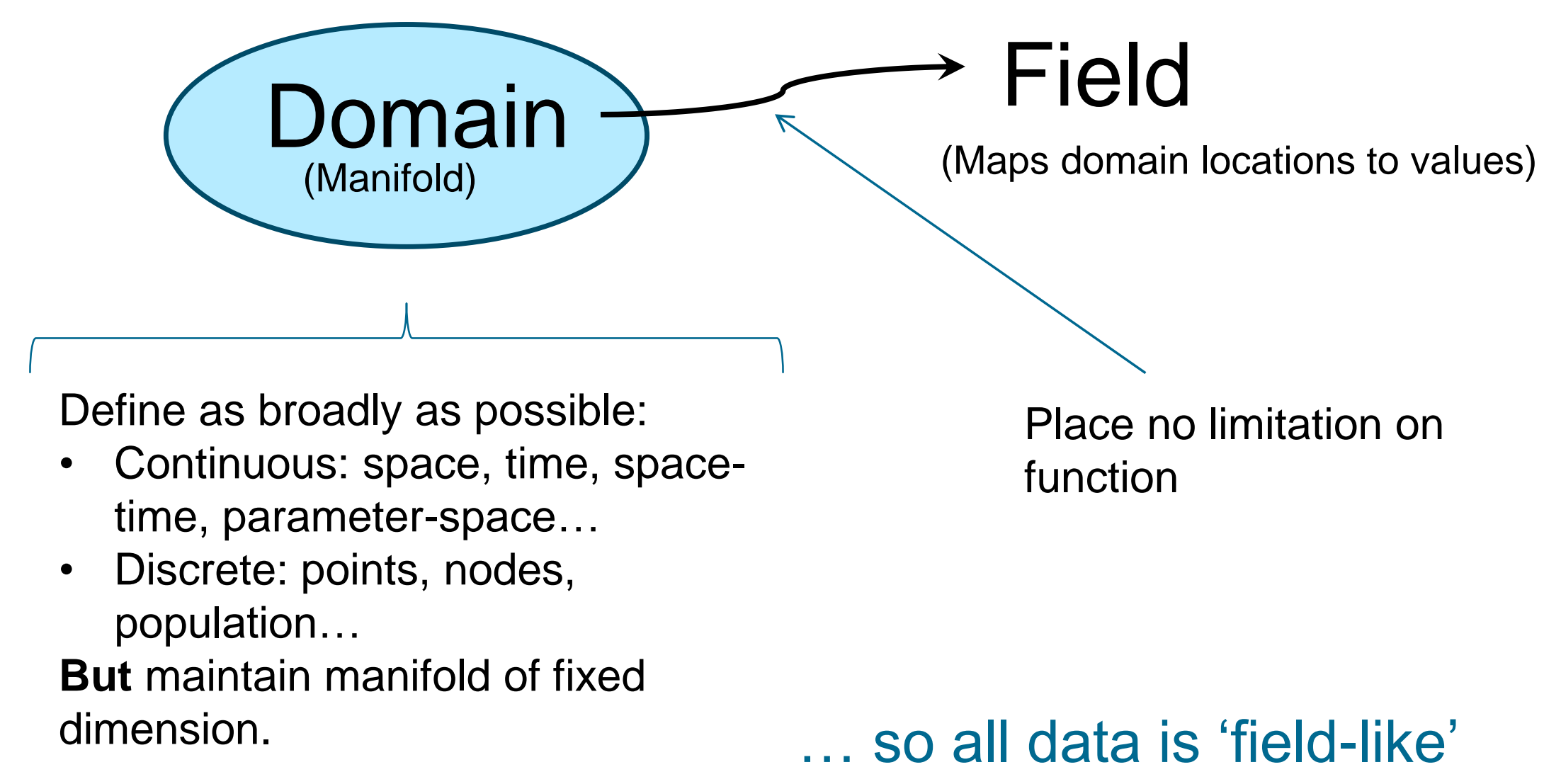




\section{FieldML Concepts: Types}

Reusable descriptions of spaces (manifolds) from which field domains are built.

- Discrete 'ensemble' type: set of unique identifiers, an

$\begin{array}{llll}1 & 2 & 3 & 4 \\ 0 & 0 & 0 & 0\end{array}$ enumeration: nodes, elements, components, time indexes, etc.

- Continuous type: $\mathrm{N}$-dimensional chart indexed by component ensemble type, possibly bounded: coordinate system $\left(\mathbb{R}^{N}\right)$, scalar domains e.g. temperature, pressure $\left(\mathbb{R}^{1}\right)$, tensor domains, etc.

- Structured type: tuple of other types with arbitrary bounds: mesh (a tuple of discrete elements and continuous element chart with bounds of the element chart given as a function of element and chart position).

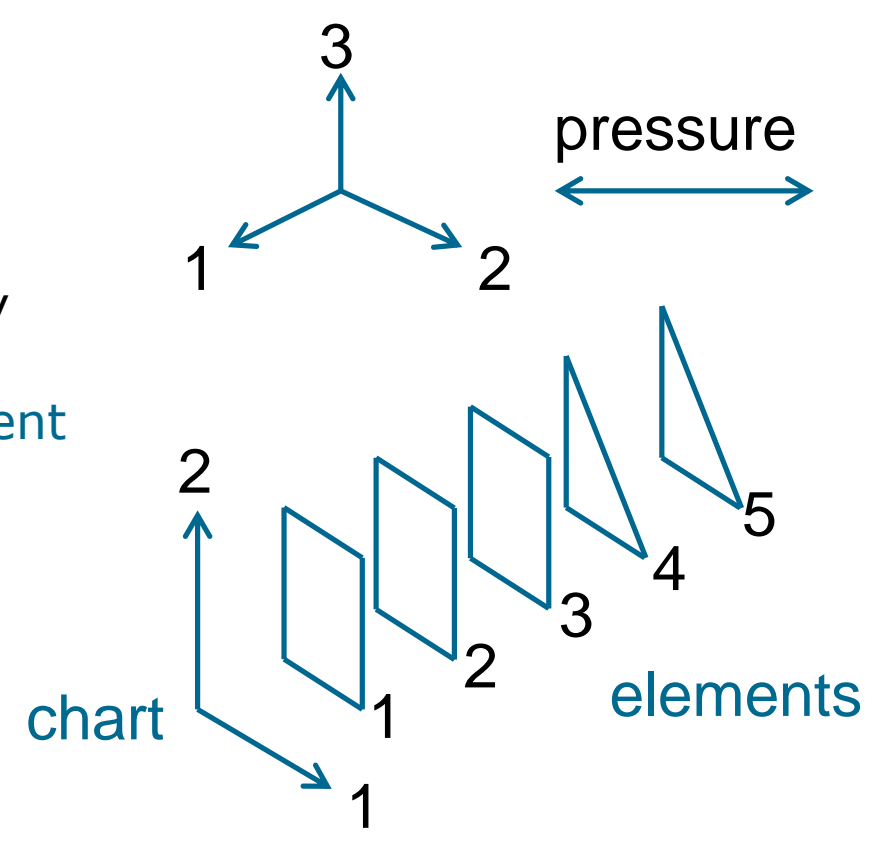




\section{FieldML Concepts: Evaluators}

- Reusable objects for building field evaluation pipelines.

- FieldML domains have no attributes except those mapped by evaluators.

Evaluators:

- ... encapsulate an operator / function,

- ... acting on values of 0 or more 'input' evaluators,

- ... to produce values of a prescribed type.

6 sub-classes of evaluators:

Abstract, Parameters, Reference, Piecewise, Aggregate and External. 


\section{Abstract Evaluator}

- The initial inputs to evaluation pipelines

$\sim$ function arguments.

- Represents a value source of prescribed type.

- Targets for binding: all abstracts must be bound to a concrete evaluator or given literal values to evaluate pipeline.

- FieldML 0.3: unbound abstract evaluators can be considered parts of field domain atlas. 


\section{Parameters Evaluator}

- Source of literal data in FieldML data model.

- Continuous or ensemble value.

- Parameters indexed by $\mathrm{N}(\geq 0)$ ensemble-valued input evaluators.

- Parameter values in external resources or inline in XML.

- Equivalent to a multi-dimensional array, dense or sparse.

- Important: can substitute any other evaluator for fixed parameters.

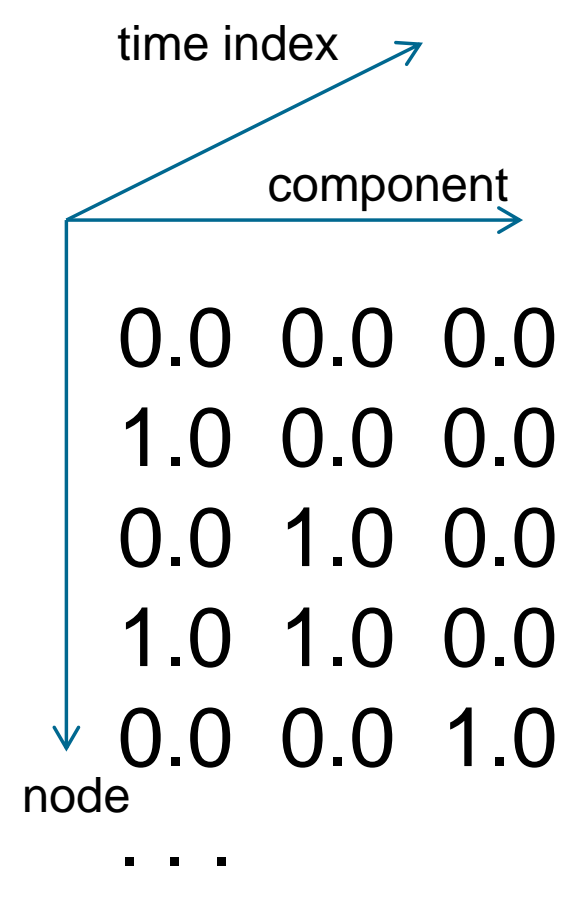




\section{Reference Evaluator}

- Evaluates a referenced evaluator, but:

- Generally binds new evaluators to one or more abstract sources of referenced evaluator to modify its behaviour.

- Similar to a function call mechanism, but arguments are functions rather than simple values.

- Only object able to reference evaluators from outside the current scope in the FieldML document. 


\section{Piecewise Evaluator}

- 'Switch' operator delegating which evaluator to use, depending on value from single ensemble-valued input evaluator.

- Often used to implement fields defined over meshes with different basis functions in each element. 


\section{Aggregate Evaluator}

- Aggregates referenced scalar evaluators over an ensemble to produce a vector valued result.

- Future: support aggregation of multiple indexes for tensor value types, aggregation of structured types. 


\section{External Evaluator}

- Named standard evaluators of agreed functionality.

- Primary extension mechanism in FieldML, usually only seen in FieldML / third-party library serialisations.

- Implementation e.g. MathML may be present, but operator may alternatively be 'understood' by name.

- Initial FieldML library supports standard element interpolation and shape functions as external evaluators. Future: support any required mathematical or other operators.

- Applications parsing FieldML must map all evaluators particularly external evaluators - to fixed objects in their data model which perform the equivalent functionality (if supported). 


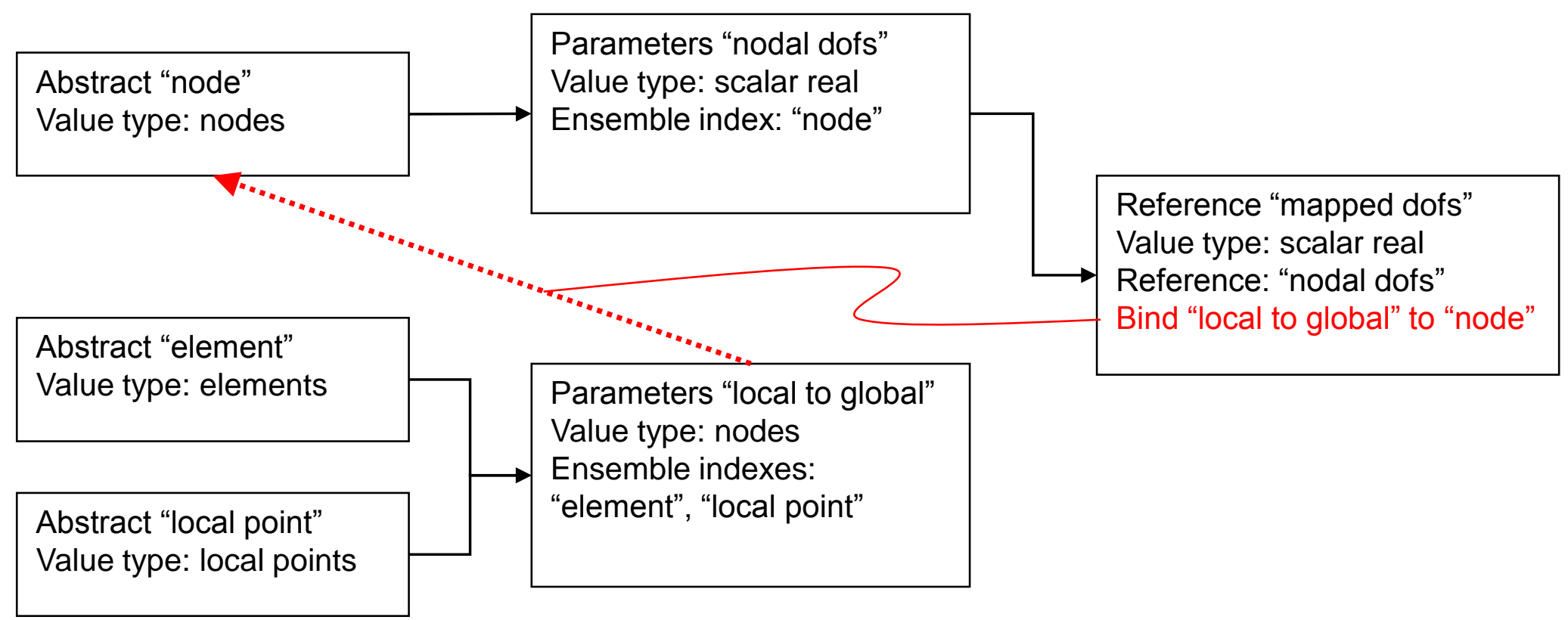

\section{Evaluator Binding}

Use in mapping 'nodal' parameters to local element. Note: binding is deeply functional, not value passing. 


\section{FieldML Features in Development}

- Regions, for building models out of sub models.

- Formal declarations of Domains and Fields c.f. interpreting evaluators as fields.

- Domains with multiple atlases, fields defined on different atlases of same domain.

- Embedding.

- Ensemble subsets and sequences for arbitrary parameter ordering.

- Hierarchical domains.

- Physical units.

- Efficient binary bulk data stores, e.g. HDF5. 


\section{FieldML benefits for large, multi-domain, multi-scale, multi-variate problems.}

- Uniform treatment of components of field domains: same basis functions + mappings usable for space, time, patient weight, population...

- Uniform treatment of structured and unstructured meshes: structured meshes differ only in using a formulaic parameter map; efficient 'semi-structured' meshes e.g. all elements of same shape.

- Uniform treatment of formerly disparate data types: Finite element interpolated variables, images, CAD geometry are all fields.

- Support for embedding, hierarchical models. 


\section{FieldML Resources}

- Website: http://www.fieldml.org/

Look out for upcoming document:

"FieldML 0.3 Concepts and Serialisation"

- FieldML API, prototypes and mock-ups: http://code.google.com/p/fieldml/

- Model repository: http://models.fieldml.org/

- Developer e-mail:

fieldml-developers@lists.sourceforge.net

- Tracker: https://tracker.physiomeproject.org/

Details are subject to change, particularly up to end of May 2011. We invite collaboration and feedback. 


\section{Acknowledgements}

- FieldML has been developed with assistance from the following organisations:
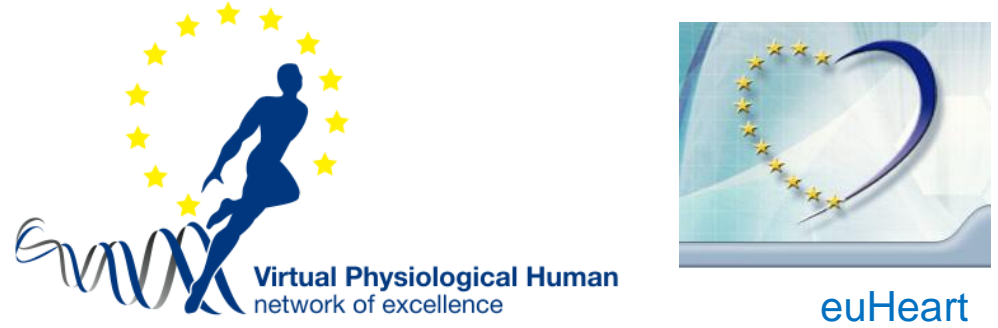

wellcometrust

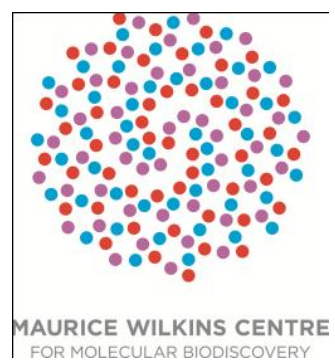

Specific grants:

- 9077 / 3622407 European Commission - FP7 VPH NoE Virtual Physiological Human Network of Excellence

- 9077 / 3625656 European Commission - FP7 International MSV Multi-scale Spatio-temporal Visualisation 


\section{Example: cube.xml}

$<$ ?xml version="1.0" encoding="ISO-8859-1"?>

$<$ fieldml version="0.3_alpha" xsi:noNamespaceSchemaLocation="Fieldml_0.3.xsd"

xmIns:xsi="http://www.w3.org/2001/XMLSchema-instance">

$<$ Region name="cube" library="library_0.3.xml">

$<$ !-- define ensemble/set of 8 entries to represent nodes $1 . .8$-->

$<$ EnsembleType name="cube.nodes">

$<$ bounds $>$

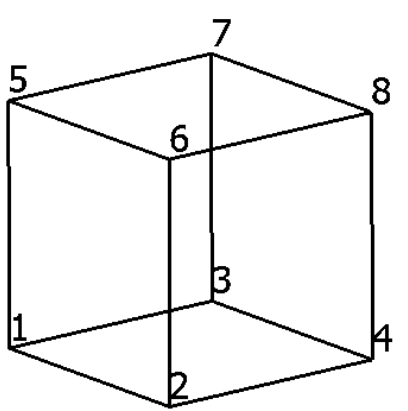

$$
</ \text { bounds }>
$$

$</$ EnsembleType $>$

<AbstractEvaluator name="cube.nodes.variable" valueType="cube.nodes"/>

<!-- declare a variable placeholder for parameters to a field template, in this case anticipated to vary with ensemble "cube.nodes" --> <AbstractEvaluator name="cube.dofs_variable.nodal" valueType="library.real.1d"/>

$<$ !-- define a 3-D mesh consisting of one element of unit cube shape. Mesh dimensionality of 3 is indicated by 3-component library ensemble "library.ensemble.xi.3d" specified for xi_component attribute -->

$<$ MeshType name="cube.mesh" xi_component="library.ensemble.xi.3d">

$<$ bounds>

$</$ bounds $>$

$<$ ContiguousEnsembleBounds valueCount="1"/>

$<$ shapes default="library.shape.cube"/>

$</$ MeshType $>$

<AbstractEvaluator name="cube.mesh.variable" valueType="cube.mesh" /> 


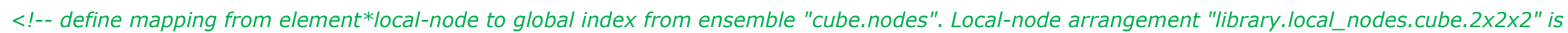
documenting in the library as being the 8 corner points of a unit cube at $x i=(0,0,0),(1,0,0),(0,1,0),(1,1,0),(0,0,1),(1,0,1),(0,1,1),(1,1,1)-->$

$<$ ParametersEvaluator name="cube.layout_2x2×2" valueType="cube.nodes" >

$<$ SemidenseData>

$<$ denseIndexes $>$

<index evaluator="library.local_nodes.cube.2x2x2.variable"/>

<index evaluator="cube.mesh.variable.element"/>

$</$ denseIndexes $>$

$<$ dataLocation $>$

$<$ inlineData>

12345678

$<$ /inlineData $>$

$</$ dataLocation $>$

$</$ SemidenseData $>$

$</$ ParametersEvaluator $>$

$<$ !-- construct a vector of nodal parameters to pass on to "cube.trilinear.interpolator" -->

$<$ AggregateEvaluator name="cube.trilinear.parameters"

valueType $=$ "library.parameters.trilinear_lagrange" $>$

<binds>

<bind_index variable="library.local_nodes.cube. $2 \times 2 \times 2$. variable"

index_number="1"/>

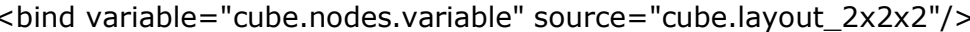

$<$ /binds $>$

<componentEvaluators default="cube.dofs_variable.nodal" />

$<$ /AggregateEvaluator $>$

\section{cube.xml 2}


<!-- parameters for the coordinate field, listing a scalar real parameter for all permutations of 3-entry

library component ensemble "library.ensemble.rc.3d" and 8-entry ensemble "cube.nodes" -->

<ParametersEvaluator name="cube.corner_coordinates" valueType="library.real.1d">

$<$ SemidenseData>

$<$ denseIndexes>

<index evaluator="library.ensemble.rc.3d.variable"/>

$<$ index evaluator="cube.nodes.variable"/>

$</$ denseIndexes $>$

$<$ dataLocation $>$

$<$ inlineData $>$

0.00 .00 .0

1.00 .00 .0

0.01 .00 .0

1.01 .00 .0

0.00 .01 .0

1.00 .01 .0

0.01 .01 .0

1.01 .01 .0

$<$ /inlineData $>$

$<$ dataLocation $>$

$</$ SemidenseData $>$

$<$ /ParametersEvaluator $>$

<!-- define evaluator returning value of library FEM basis evaluator "library.fem.trilinear_lagrange" at the element chart location of mesh type

"cube.mesh" (denoted by "cube.mesh.xi") and using parameters from evaluator "cube.trilinear.parameters". -->

$<$ ReferenceEvaluator name="cube.trilinear.interpolator"

evaluator="library.fem.trilinear_lagrange"

valueType="library.real. $1 \mathrm{~d} ">$

$<$ binds $>$

$<$ bind variable $=$ "library.xi.3d.variable" source="cube.mesh.variable.xi"/>

$<$ bind variable $=$ "library. parameters.trilinear_lagrange.variable"

source="cube.trilinear. parameters"/>

$</$ binds $>$

$</$ ReferenceEvaluator $>$

cube.xml 3 


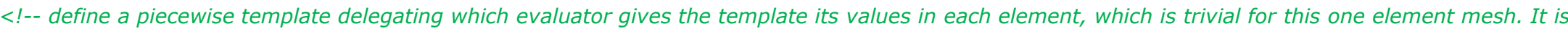
a template for a field defined over the mesh represented by "cube.mesh.variable", with the unbound parameter source "cube.dofs_variable.nodal"

inherited from delegate evaluator "cube.trilinear.interpolator" -->

<PiecewiseEvaluator name="cube.template.trilinear" valueType="library.real.1d">

<binds>

<bind_index variable="cube.mesh.variable.element" index_number="1" />

$</$ binds $>$

$<$ elementEvaluators>

$<$ element number="1" evaluator="cube.trilinear.interpolator"/>

$<$ /elementEvaluators $>$

$</$ PiecewiseEvaluator $>$

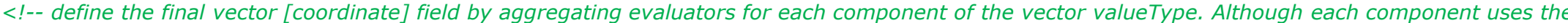

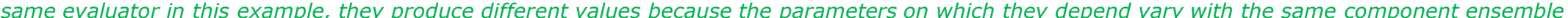
("library.ensemble.rc.3d") which the library documents as indexing this field's valueType (continuous type "library.coordinates.rc.3d"). Hence only one binding is required to set the parameter source for this field -->

$<$ AggregateEvaluator name="cube.coordinates" valueType="library.coordinates.rc.3d">

<binds>

<bind_index variable="library.ensemble.rc.3d.variable" index_number="1" />

<bind variable="cube.dofs_variable.nodal" source="cube.corner_coordinates"/>

$</$ binds $>$

$<$ componentEvaluators $>$

<component number="1" evaluator="cube.template.trilinear"/>

$<$ component number="2" evaluator="cube.template.trilinear"/>

<component number="3" evaluator="cube.template.trilinear"/>

$</$ componentEvaluators $>$

$</$ AggregateEvaluator $>$

$</$ Region $>$

$</$ fieldml $>$

\section{cube.xml 4}

